\title{
A prospective study of visual function and quality of life following PDT in patients with wet age related macular degeneration
}

\author{
A M Armbrecht, P A Aspinall, B Dhillon
}

Br J Ophthalmol 2004;88:1270-1273. doi: 10.1136/bjo.2003.038604

\begin{abstract}
Aims: (1) A prospective study to assess visual function measures and quality of life (QoL) in patients with wet age related macular degeneration (AMD) treated with photodynamic therapy (PDT). (2) To assess if PDT prevents severe visual loss (loss of six or more lines of distance visual acuity) in the treated eye.

Methods: 48 of 51 recruited patients with predominantly classic subfoveal choroidal neovascularisation (CNV) secondary AMD who were treated with PDT were followed up for 1 year. Assessment included distance and near visual acuity, contrast sensitivity, vision related quality of life and fluorescein angiography. Photodynamic therapy using Visudyne was carried out according to standard protocol. Patients were followed up every 3 months and treatment repeated if there was significant leakage from CNV.

Results: At the 12 month follow up, $71 \%(n=34)$ of the patients lost less than three lines of best corrected distance visual acuity. Although there were significant decreases in some of the QoL items tested, patients were significantly less anxious and more independent outdoors at the 12 month follow up.

Conclusion: This study is in keeping with published literature with PDT preventing severe visual loss in two thirds of treated patients with predominantly classic CNV.
\end{abstract}

A ge related macular degeneration (AMD) is the main cause of blindness in the Western world in patients over the age of 65 years. Choroidal neovascularisation (CNV) accounts for approximately $90 \%$ of cases of severe visual loss in patients with AMD.

At present, there are no satisfactory treatments for this condition. ${ }^{1}$ Laser photocoagulation is useful in only a minority of patients with wet AMD. ${ }^{2-4}$ Research in different treatments is ongoing. ${ }^{56}$

Photodynamic therapy (PDT) has been recently introduced as an alternative treatment for CNV and extensive preclinical and clinical research data are available. ${ }^{7-19}$

The results of two randomised clinical trials reported that PDT using verteporfin could safely reduce the risk of vision loss in patients with subfoveal CNV secondary to AMD. ${ }^{13} 1617$

The aim of this study was to assess visual function and quality of life of patients with CNV secondary to macular degeneration treated with PDT.

\section{PATIENTS AND METHODS}

This study was carried out at Princess Alexandra Eye Pavilion in Edinburgh. Patients were recruited between October 2000 and April 2002 and follow up is still ongoing.
Inclusion criteria: patients who, on fluorescein angiography, had evidence of CNV with at least 50\% classic component, no greater than $5400 \mu \mathrm{m}$, secondary to AMD with visual acuity no worse than 6/36 in the study eye and who were willing to participate in the study.

Exclusion criteria: (1) any significant ocular disease different from CNV secondary to AMD; (2) inability to obtain photographs and fluorescein angiography; (3) inability to understand what is involved in the treatment and consent; (4) presence of liver disease, porphyria, and/or unstable heart condition.

Patients were assessed at baseline and every 3 months thereafter. At each visit the following assessment was carried out: (1) history of present illness or change in eye condition if applicable; (2) past medical and ocular history if applicable; (3) assessment of sitting blood pressure, pulse and calculation of body surface area; (4) best corrected visual acuity with refraction protocol for distance and near using logMAR Bailey-Lovie charts; (5) assessment of contrast sensitivity using Pelli-Robson charts; (6) slit lamp and retinal examination under dilated pupil; (7) assessment of quality of life (QoL) using a previously designed questionnaire that includes the VF- $14,{ }^{20}$ and other questions regarding response to the visual problem and mood (at baseline and 1 year follow up only). The questionnaire was validated for a previous study. ${ }^{21}$ Scales used in the questionnaire are illustrated in the appendix; (8) fluorescein angiography using Topcon V 1.52 digital fundus camera if the last one was done more than 1 week ago, with documentation of size and location of the lesion and percentage of classic component; (9) signed informed consent for both treatment and study participation; (10) the family doctor was informed of the patient's participation in the study.

Photodynamic therapy was carried out according to a standard protocol: $6 \mathrm{mg}$ of verteporfin per square metre of body surface area was infused over a period of 10 minutes. Then, 15 minutes after starting the infusion, the whole lesion was treated with a laser light at $689 \mathrm{~nm}$ delivering $50 \mathrm{~J} / \mathrm{cm}^{2}$ at an intensity of $600 \mathrm{~mW} / \mathrm{cm}^{2}$ over a period of 83 seconds. ${ }^{13}$

After treatment, a leaflet with instructions and dark sunglasses were provided, as well as a bracelet stating the name of the patient, date and time of treatment, and contact number in case of an emergency.

For the statistical analysis SPSS 10 was used. For the assessment of change over time in the same patients the Wilcoxon signed rank test was carried out.

Abbreviations: $A M D$, age related macular degeneration; $C N V$, choroidal neovascularisation; PDT, photodynamic therapy; QoL, quality of life 
Table 1 Mean (SD) values at baseline and 1 year follow up for the whole cohort (48 patients)

\begin{tabular}{|c|c|c|c|c|c|}
\hline & \multicolumn{2}{|c|}{ Baseline } & \multicolumn{2}{|l|}{1 year } & \multirow{2}{*}{$\begin{array}{l}\text { p Values for change } \\
\text { (Wilcoxon SRT) }\end{array}$} \\
\hline & Mean & SD & Mean & SD & \\
\hline \multicolumn{6}{|l|}{ Visual function measures and VF-14 items: } \\
\hline $\begin{array}{l}\text { Visual function tests } \\
\text { Distance VA (study eye) } \\
\text { Near VA (study eye) } \\
\text { Contrast sensitivity (study eye) } \\
\text { CNV largest linear dimension ( } \mu \mathrm{m} \text { ) } \\
\text { General questions on vision } \\
\text { Treatment worthwhile } \\
\text { Trouble with vision } \\
\text { Satisfaction with vision } \\
\text { Change in vision } \\
\text { VF-14 questionnaire } \\
\text { Read small print } \\
\text { Read newspaper or book } \\
\text { Large print books } \\
\text { Recognise people close } \\
\text { See steps/kerbs } \\
\text { Read street signs } \\
\text { Do fine handwork } \\
\text { Fill forms or cheques } \\
\text { Cooking } \\
\text { Watching TV } \\
\text { Crossing roads } \\
\text { Recognise faces across the street } \\
\text { Read bus numbers } \\
\text { Social activities } \\
\text { Getting about indoors } \\
\text { Hobbies } \\
\text { Total VF-14 score }\end{array}$ & $\begin{array}{l}0.61 \\
0.92 \\
1.14 \\
3094 \\
\\
\text { NA } \\
2.3 \\
2.2 \\
1.9 \\
\\
1.4 \\
1.7 \\
1.8 \\
3.5 \\
3.4 \\
3.0 \\
1.5 \\
2.5 \\
3.2 \\
2.4 \\
3.0 \\
1.9 \\
2.6 \\
3.1 \\
3.8 \\
2 \\
68\end{array}$ & $\begin{array}{ll} & 0.19 \\
0.28 \\
0.25 \\
1201 \\
\\
4.2 \\
1.0 \\
1.0 \\
1.0 \\
\\
1.7 \\
1.7 \\
1.7 \\
0.97 \\
0.74 \\
1.4 \\
1.6 \\
1.5 \\
1.2 \\
1.1 \\
1.2 \\
1.7 \\
1.5 \\
1.4 \\
0.39 \\
1.7 \\
66\end{array}$ & $\begin{array}{l}0.80 \\
1.1 \\
1.11 \\
4088 \\
\\
0.98 \\
2.1 \\
2.7 \\
2.8 \\
\\
1.2 \\
1.5 \\
1.3 \\
3.3 \\
3.3 \\
2.1 \\
0.89 \\
1.9 \\
3.3 \\
2.5 \\
2.3 \\
1.2 \\
1.9 \\
3.1 \\
3.8 \\
2.1 \\
25\end{array}$ & $\begin{array}{l}0.30 \\
0.35 \\
0.35 \\
1532 \\
\\
\text { NA } \\
0.94 \\
1.2 \\
0.87 \\
1.6 \\
1.7 \\
1.7 \\
1.1 \\
0.90 \\
1.7 \\
1.4 \\
1.6 \\
0.97 \\
1.3 \\
1.4 \\
1.6 \\
1.7 \\
1.2 \\
0.41 \\
1.7 \\
0.11\end{array}$ & $\begin{array}{l}<0.01 \downarrow \\
<0.02 \downarrow \\
0.31 \\
<0.01 \downarrow \\
\\
0.13 \\
0.09 \\
<0.01 \uparrow \\
0.79 \\
0.38 \\
0.53 \\
0.02 \downarrow \\
0.79 \\
<0.01 \downarrow \\
0.24 \\
<0.01 \downarrow \\
0.85 \\
0.97 \\
<0.01 \downarrow \\
<0.01 \downarrow \\
\mathbf{0 . 0 2} \downarrow \\
0.17 \\
0.71 \\
0.38\end{array}$ \\
\hline \multicolumn{6}{|l|}{ Questions on mood: } \\
\hline $\begin{array}{l}\text { Nervous person } \\
\text { Felt downhearted/low } \\
\text { Been happy person } \\
\text { Irritated because of AMD } \\
\text { Anxious because of AMD }\end{array}$ & $\begin{array}{l}4.2 \\
3.9 \\
3.7 \\
3.7 \\
3.4\end{array}$ & $\begin{array}{l}1.1 \\
1.0 \\
0.94 \\
1.2 \\
1.2\end{array}$ & $\begin{array}{l}4.2 \\
4.2 \\
4.0 \\
3.7 \\
4.0\end{array}$ & $\begin{array}{l}1.1 \\
0.82 \\
0.88 \\
1.3 \\
1.2\end{array}$ & $\begin{array}{l}0.28 \\
0.23 \\
0.08 \\
0.75 \\
<0.01 \uparrow\end{array}$ \\
\hline \multicolumn{6}{|l|}{ Response to visual problem: } \\
\hline $\begin{array}{l}\text { Stay at home most of the time } \\
\text { Don't need lot help from others } \\
\text { Worry about doing embarrassing things } \\
\text { Control over what you want to do }\end{array}$ & $\begin{array}{l}2.7 \\
3.8 \\
3.5 \\
4.0\end{array}$ & $\begin{array}{l}1.6 \\
1.3 \\
1.6 \\
1.3\end{array}$ & $\begin{array}{l}3.1 \\
3.4 \\
3.6 \\
4.0\end{array}$ & $\begin{array}{l}1.5 \\
1.3 \\
1.4 \\
0.93\end{array}$ & $\begin{array}{l}0.04 \uparrow \\
0.17 \\
0.92 \\
0.93\end{array}$ \\
\hline
\end{tabular}

\section{RESULTS}

Fifty one patients with predominantly classic CNV secondary to AMD with visual acuity of $6 / 36$ or better in the study eye at baseline were recruited. Of these, three withdrew from the study, one because of no response to treatment at second follow up visit and two because of poor general health which prevented the patients from complying with 3 monthly follow up visits and angiograms. Of the 48 remaining patients $23(48 \%)$ were male and $25(52 \%)$ were female. Mean age was 72 years ranging from $51-87$ years. In 19 $(40 \%)$ patients the study eye was the first eye developing wet AMD - that is, the fellow eye either had mild age related maculopathy (ARM) or moderate dry ARM. In the remaining 29 patients $(60 \%)$ the study eye was the second eye involved-that is, the fellow eye either had AMD (25 patients) or other disease causing severe visual loss or blindness (previous retinal detachment in one, childhood trauma in two, and profound amblyopia in one).

All patients in this cohort had predominantly classic subfoveal CNV. Of these, 19 (39\%) were purely classic lesions. On average patients needed 3.1 treatments in the first year. At 1 year follow up, distance visual acuity improved at least one line in $11(23 \%)$ eyes, 23 (48\%) eyes lost three lines or less of baseline distance visual acuity, and $14(29 \%)$ eyes lost more than three lines of vision. In total, 34
(71\%) of the patients lost three lines or less of distance vision and on average the whole cohort of patients lost two lines of distance visual acuity.

Table 1 shows mean values for visual function tests and quality of life items at baseline and 12 month follow up together with $p$ values for change over time (Wilcoxon signed rank test) for the whole cohort. Negative significant values (that is, poorer function) are highlighted in bold with downward arrow next to the value. Positive significant values (that is, better function) are highlighted in bold with upward arrow next to the value. Values for visual acuity measurements are expressed in $\log$ MAR units and for contrast sensitivity in log units.

Table 1 shows that there were significant losses both in visual function tests and visual related QoL issues. Nevertheless, there were also significant improvements in aspects such as anxiety related to AMD, independence outdoors, and subjectively patients felt that their vision had remained stable and considered the treatment as better than "quite worthwhile."

In this study all recruited patients were treated and there is no control group available for comparison. Nevertheless, the fellow non-treated eye in the group of patients with second eye involvement could serve as control for the assessment of the behaviour of visual function tests in time. Table 2 shows 
Table 2 Mean values for visual function tests and CNV size for study eye (treated) and fellow eye (untreated) in patients with second eye involvement $(n=29)$

\begin{tabular}{|c|c|c|c|c|c|}
\hline & \multicolumn{2}{|c|}{ Study eye } & \multicolumn{2}{|c|}{ Fellow eye } & \multirow{2}{*}{$\begin{array}{l}\text { p Values } \\
\text { (Wilcoxon SRT) }\end{array}$} \\
\hline & Mean & SD & Mean & SD & \\
\hline Distance VA at 12 months & 0.86 & 0.25 & 1.27 & 0.35 & $<0.01$ \\
\hline Near VA at 12 months & 1.1 & 0.31 & 1.5 & 0.32 & $<0.01$ \\
\hline Contrast sensitivity at 12 months & 1.16 & 0.34 & 0.84 & 0.43 & $<0.01$ \\
\hline CNV size at 12 months & 4754 & 1194 & 6809 & 2004 & $<0.01$ \\
\hline
\end{tabular}

that the treated eye continues to be significantly better at the 12 month follow up.

\section{Side effects}

Three patients developed back pain during the infusion, which recurred on repeated treatments. One patient developed a subretinal bleed after treatment, which resolved in time and visual acuity returned to pre-bleeding levels.

\section{DISCUSSION}

The results of this study are consistent with those of the TAP trials with $71 \%$ of our patients losing three lines or fewer of distance vision at the 12 month follow up. The TAP trials demonstrated that $69 \%$ of the patients with predominantly classic CNV treated with PDT lost three lines or fewer of distance visual acuity at 12 month follow up. ${ }^{13}$ PDT is a treatment intended to prevent severe visual loss (loss of six or more lines of distance visual acuity). If left untreated subfoveal classic lesions lead to severe visual loss (visual acuity of $6 / 60$ or worse) in $90 \%$ of the patients ${ }^{2}$ with most of the visual loss occurring in the first year. ${ }^{22}$

Vision related QoL is directly linked with the severity of visual loss and whether one or both eyes are affected. If vision is poor, vision related quality of life is also poor. The results regarding QoL issues for the patients in this study reflect what the treatment achieves-that is, prevention of severe visual loss, but still there is moderate visual loss.

It is not clear why there was significant improvement in some QoL aspects in the presence of progressive visual loss. This finding is not necessarily related to an effect of the treatment but may just reflect that at 12 month follow up patients were better adapted to their visual disability.

This study suggests that although PDT is useful in the management of exudative AMD, its usefulness is limited by the fact that patients may still lose vision even when treated and in only a minority of patients is there small improvement in visual function. It is therefore clear that there is an increasing need for further research into understanding the aetiology of ARM/AMD and factors that modify its behaviour and progression.

Involving patients in therapeutic decision making is a major objective of Quality Improvement Scotland and the present data suggest that patients did consider the treatment more than "quite worthwhile". Patients in this study understood the limitations of PDT and setting patients' expectations is an important prerogative when treating patients with PDT.

\section{CONCLUSION}

This study shows that while significant deterioration in vision occurs in the treated eye, PDT does prevent severe visual loss in approximately two thirds of treated patients with predominantly classic subfoveal CNV secondary to AMD and its use is therefore justified in these patients. This study also confirms the clinical perception that vision related quality of life does not improve unless a treatment is able to improve or restore vision in a significant number of treated patients.

\section{ACKNOWLEDGEMENTS}

This study was sponsored by the Scottish National Institute for the War Blinded. We are also grateful to the photography department at Princess Alexandra Eye Pavilion, Edinburgh.

\section{Authors' affiliations}

A M Armbrecht, B Dhillon, Princess Alexandra Eye Pavilion, Chalmers Street, Edinburgh EH3 9HA, UK

P A Aspinall, Heriot-Watt University, Edinburgh EH14 4AS, UK

Correspondence to: A M Armbrecht, Princess Alexandra Eye Pavilion, Chalmers Street, Edinburgh EH3 9HA, UK; amarmbrech+@yahoo.com

Accepted for publication 2 March 2004

\section{APPENDIX}

\section{SCALES USED IN THE QUESTIONNAIRE}

\section{General questions}

Do you consider PDT treatment you've received worthwhile?

- 1 Not at all

- 2 Not worthwhile

- 3 Worthwhile

- 4 Quite worthwhile

- 5 Very worthwhile

How much trouble do you have presently with your vision?

- 1 A great deal

- 2 A moderate amount

- 3 A little

- 4 None

How satisfied are you presently with your vision?

- 1 Not at all satisfied

- 2 Not satisfied

- 3 Satisfied

- 4 Quite satisfied

- 5 Very satisfied

How would you describe the change if any that you have noticed in your vision over the past year?

- 1 Very much worse

- 2 Slightly worse

- 3 The same

- 4 Slightly better

- 5 Very much better 
VF- 14

Do you have any difficulty even with glasses in doing any of the following activities? If yes how much difficulty do you currently have? NA meaning that the question is not applicable to you or you do not do it for reasons other than your vision.

- 0 Unable to do

- 1 A great deal of difficulty

- 2 A moderate amount of difficulty

- 3 A little

- 4 No difficulty

- 99 Not applicable

Questions of mood

How much of the time over the last 4 weeks have you been?

(a) nervous, (b) downhearted or low, (c) irritated with eye condition, and (d) anxious because of eye condition:

- 1 All the time

- 2 Most of the time

- 3 A good bit of the time

- 4 Some of the time

- 5 None of the time

For "Been a happy person":

- 5 All the time

- 4 Most of the time

- 3 A good bit of the time

- 2 Some of the time

- 1 None of the time

\section{QUESTIONS IN RESPONSE TO VISUAL PROBLEM}

For each of the following statements please tell me if you agree or disagree with them

(a) Stay at home most of the time and (b) worry about doing things that will embarrass myself or others.

- 1 Strongly agree

- 2 Agree

- 3 Neutral

- 4 Disagree

- 5 Strongly disagree

- 99 Don't know

(a) I do not need a lot of help from others and (b) I have control over what I want to do

- 5 Strongly agree

- 4 Agree

- 3 Neutral

- 2 Disagree

- 1 Strongly disagree

- 99 Don't know

\section{REFERENCES}

1 Klein R. Epidemiology. In: Berger JW, Fine SL, Maguire MG, eds. Age-related macular degeneration. 1st ed. Chapter 3. St Lovis: Mosby, 1999:1.

2 Macular Photocoagulation Study Group. Laser photocoagulation of subfoveal neovascular lesions of age-related macular degeneration. Updated findings from two clinical trials. Arch Ophthalmol 1993;111:1200-9.

3 Macular Photocoagulation Study Group. Visual outcome after laser photocoagulation for subfoveal choroidal neovascularization secondary to age-related macular degeneration. The influence of initial lesion size and initial visual acuity. Arch Ophthalmol 1994;112:480-8.

4 Macular Photocoagulation Study Group. Laser photocoagulation for juxtafoveal choroidal neovascularization. Five-year results from randomized clinical trials. Arch Ophthalmol 1994;112:500-9.

5 Ciulla TA, Danis RP, Harris A. Age-related macular degeneration: a review of experimental treatments. Surv Ophthalmol 1998;43:134-46.

6 Hooper CY, Guymer RH. New treatments in age-related macular degeneration. Clin Experiment Ophthalmol 2003;31:376-91.

7 Schmidt-Erfurth U, Hasan T, Gragoudas ES, et al. Vascular targeting in photodynamic occlusion of subretinal vessels. Ophthalmology 1994:101:1953-61.

8 Hussain D, Miller JW, Michaud N, et al. Intravenous infusion of liposomal benzoporphyrin derivative for photodynamic therapy of experimental choroidal neovascularization. Arch Ophthalmol 1996;1 14:978-85.

9 Kramer M, Miller JW, Michaud N, et al. Liposomal benzoporphyrin derivative verteporfin photodynamic therapy. Selective treatment of choroidal neovascularization in monkeys. Ophthalmology 1996; 103:427-38.

10 Hussain D, Kramer M, Kenny AG, et al. Effects of photodynamic therapy using verteporfin on experimental choroidal neovascularization and normal retina and choroid up to 7 weeks after treatment. Invest Ophthalmol Vis Sci 1999;40:2322-31.

11 Miller JW, Schmidt-Erfurth U, Sickenberg M, et al. Photodynamic therapy with verteporfin for choroidal neovascularization caused by age-related macular degeneration. Results of a single treatment in phase 1 and 2 study. Arch Ophthalmol 1999;117:1161-73.

12 Schmidt-Erfurth U, Miller JW, Sickenberg M, et al. Photodynamic therapy with verteporfin for choroidal neovascularization caused by age-related macular degeneration. Results of re-treatments in a phase 1 and 2 study. Arch Ophthalmol 1999;117:1177-87.

13 Treatment of Age-related Macular Degeneration with Photodynamic Therapy (TAP) Study Group. Photodynamic therapy of subfoveal choroidal neovascularization in age-related macular degeneration with verteporfin. Arch Ophthalmol 1999;117:1329-45.

14 Sickenber M, Schmidt-Erfurth U, Miller JW, et al. A preliminary study of photodynamic therapy using verteporfin for choroidal neovascularization in pathologic myopia, ocular histoplasmosis syndrome, angioid streaks and idiopathic causes. Arch Ophthalmol 2000;117:327-36

15 Schmidt-Erfurth U, Hasan T. Mechanisms of action of photodynamic therapy with verteporfin for the treatment of age-related macular degeneration. Surv Ophthalmol 2000;45:195-214.

16 TAP Study Group. Photodynamic therapy of subfoveal choroidal neovascularization in age-related macular degeneration using verteporfin (Visudyne): two-year results of 2 randomized clinical trials. TAP report No. 2. Arch Ophthalmol 2001;119:198-207.

17 TAP Study Group. Verteporfin therapy for subfoveal choroidal neovascularization in age-related macular degeneration. Three-year results of an open-label extension of 2 randomized clinical trials. TAP report No 5. Arch Ophthalmol 2002;120:1307-14.

18 Schmidt-Erfurth U, Michels S, Barbazetto I, et al. Photodynamic effects on choroidal neovascularization and physiological choroid. Invest Ophthalmol Vis Sci 2002;43:830-41.

19 Parodi MB, Da Pozzo S, Ravalico G. Angiographic features after photodynamic therapy for choroidal neovascularization in age-related macular degeneration and pathological myopia. $\mathrm{Br} J$ Ophthalmol 2003;87:177-83.

20 Steinberg EP, Tielsh JM, Shein OD, et al. The VF-14. An index of functional impairment in patients with cataract. Arch Ophthalmol $1994 ; 112: 630-8$

21 Armbrecht AM, Findlay C, Aspinall PA, et al. Cataract surgery in patients with age-related macular degeneration. One year outcomes. J Cataract Refract Surg 2003;29:686-93.

22 Maguire M. Natural history. In: Berger JW, Fine SL, Maguire M, eds. Agerelated macular degeneration. 1st ed. Chapter 2. St Louis: Mosby, 1999:26. 